\title{
Las implicancias de la circularidad de la comprensión en el desarrollo del discurso cientifico: un estudio acerca de la concep- ción heideggeriana de los tres niveles de la precomprensión.
}

\section{The implications of the circularity of understan- ding in the development of scientific discourse: A study on the heideggerian conception of the three levels of pre-understanding.}

\author{
LUCIANO MASCARÓ \\ (CONICET, ANCBA-CEF)
}

Recibido: 21/04/15

Aceptado: 27/04/15

\section{RESUMEN}

En este estudio nos proponemos investigar la influencia de la dimensión retornante de todo comprender en la génesis existencial de la ciencia en general. En un trabajo de unificación de temáticas dispersas a lo largo del período de Marburgo, intentaremos poner de manifiesto la triple estructura del comprender previo, y el modo en que las ciencias ónticas interpretan el carácter circular del comprender e intentan -en vano- escapar de él. La investigación revelará que el carácter retornante de la comprensión define no sólo las tareas fácticas de una ciencia, sino también su perfil histórico y tradicional, su posibilidad de crisis y desarrollo, y su misma génesis existencial.

HEIDEGGER, CÍRCULO HERMENÉUTICO, CIENCIA, PRE-COMPRENSIÓN 


\section{ABSTRACT}

In this study, we attempt to analyze the influence of the returning character of every form of understanding in the existential genesis of science in general. In a task that requires the articulation of subjects which appear in a disperse manner during the Marburg-period, we will attempt to manifest the triple structure of the pre-understanding, and the way in which ontic sciences interpret the circular character of understanding, and struggle -in vain- to abandon it. Our research will reveal that the returning character of understanding defines not only the factic tasks of a science, but also its historical and traditional profile, its likelyhood for crisis and development, and its existential genesis in itself.

KEYWORDS

HEIDEGGER, HERMENEUTIC CIRCLE, SCIENCE, PRE-UNDERSTANDING

\section{INTRODUCCIÓN}

El PROBLEMA DEL CÍRCULO HERMENÉUTICO ocupa un lugar central dentro de las consideraciones heideggerianas acerca de la estructura de la comprensión como existenciario a lo largo del período de Marburgo (lecciones impartidas entre 1923 y 1927). Este período del pensar de Heidegger se ubica en una coyuntura histórica peculiar, una situación de las ciencias ónticas denominada crisis de fundamentos [Grundlagenkrisis] (Heidegger 2006a, p.32). Es en este contexto que los estudios en torno a la estructura circular de la comprensión se derraman sobre el fenómeno puntual de la ciencia, que ya había sido definido como una actitud o comportamiento [Verhaltung] del Dasein en el mundo, en la así llamada «noción existencial de la ciencia» [Existenziale Begriff der Wissenschaft] (Ibid., p.373). Con todo, las problemáticas asociadas específicamente a la constitución ontológica de las ciencias aparecen en las lecciones de Marburgo de manera dispersa y desarticulada.

En primer lugar, nos proponemos reconstruir y explicitar el modo de recepción del carácter circular del comprender por parte de las disciplinas científicas ónticas, esto es, la consideración de la circularidad como contraria a las tradicionales exigencias de rigurosidad científica. Según la lectura de Heidegger, la ciencia percibe dos riesgos fundamentales en la circularidad de la comprensión 1) el de introducir consideraciones subjetivas en las investigaciones y 2) el de no ser capaz de expandir el conocimiento, debido al movimiento de regreso sobre lo mismo.

Posteriormente, en una lectura más profunda, analizaremos la influencia de la dimensión retornante de todo comprender en la génesis existencial de la ciencia en general. Por último intentaremos poner de manifiesto, en un trabajo de unificación de temáticas dispersas, que este carácter retornante y sedimentado del comprender no trae aparejada ninguna de aquellas temidas consecuencias, 
que la postura que así lo afirma realiza una lectura sesgada de la comprensión, y finalmente, que el carácter circular de la comprensión se haya en la misma base de la constitución de todo saber científico.

\section{El Círculo del COMPRender}

Podemos encontrar una exposición del círculo del comprender [Der Zirkel im Verstehen] en el capítulo quinto de Ser y Tiempo, dedicado a la aclaración de la constitución existencial del Ahí. En este contexto, Heidegger se detiene en la consideración de la estructura ontológica del sentido [Sinn], esto es, el horizonte de comprensibilidad de algo.

El sentido se configura por la unidad de tres fenómenos cooriginarios, «tres presuposiciones que caracterizan la situación hermenéutica» (Kockelmans, 1985, p.86), tres estructuras desde las cuales se realiza todo interpretar: el haber previo (Vorhabe), la forma previa de ver (Vorsicht), y la forma previa de concebir (Vorgriff). Estas tres dimensiones cumplen la función de horizonte desde el cual se parcializan los aspectos que incumben a la existencia que comprende. La Vorhabe nombra la disponibilidad previa de la totalidad respeccional como trasfondo para cualquier modo del comprender; la Vorsicht nombra la orientación previa desde donde se incorpora lo comprendido, un cierto punto de vista que recorta lo pre-comprendido, destacando en ello tales o cuales aspectos; La Vorgriff hace referencia al aparato conceptual, operativo de antemano por medio del cual ocurre toda apropiación interpretativa de lo comprendido.

Estas tres dimensiones representan un depósito permanente y operante, un trasfondo que subyace a cualquier modalidad de la comprensión que se dirige al mundo. Esta serie de adquisiciones previas e inexplícitas, surgidas del habitual estar-en-el-mundo y del estar-con-otros [Mitsein] conforman la estructura total del horizonte de comprensibilidad. Es desde esta estructura de donde serán tomados los sentidos adecuados a través de los cuales se comprende lo que viene a la presencia.

Sin embargo, un acto de comprensión (cualquiera sea su modalidad) ${ }^{1}$ no se limita a tomar el sentido de lo presente desde el depósito conformado por el trasfondo de sentido. Según se observa al estudiar el fenómeno de la aperturidad [Erschlossenheit], a la existencia le pertenece esencialmente un desplegarse y proyectar, un permanente quedar dirigida hacia el mundo y los otros. Este proyecto fáctico del Dasein matiza los entes que vienen a la presencia, el estado de ánimo, la actitud inquisitiva de la contemplación científica, la visión artística, el interés de la operación manual, cada una de estas modalidades preparan al Dasein para recibir lo presente como definido ciertos aspectos.

1 Teorética, artística, ocupacional, etc. 
Ahora bien, la aperturidad y su proyectar, por una parte, y el trasfondo de sentido con sus adquisiciones previas, por la otra, funcionan de manera siempre conjunta. Todo comprender se realiza desde un trasfondo de entes, objetos, existencias y situaciones alguna vez aprehendidas, aunque inexplícitamente. Sobre ese horizonte trabajará la aperturidad, sirviéndose de la comprensibilidad previa y habitual, para adaptarla a las nuevas situaciones. Es en este sentido que se ha afirmado que todo comprender es, en cierto modo, un retornar. He aquí la naturaleza del círculo del comprender. «Toda interpretación que haya de aportar comprensión debe haber comprendido ya lo que en ella se ha de interpretar» (Heidegger 2006a, p.176)

Lo dicho puede formularse de otra manera: Siempre se comprende «algo en tanto algo» [Etwas als Etwas] (Ibid., p.172). El «en tanto qué» [Als] nombra la estructura hermenéutica de la existencia. Al comprender, la existencia sale de sí para regresar sobre sí. ${ }^{2}$

La comprensión parte en busca de lo novedoso, lo cual es incorporado y articulado (mediante la interpretación [Auslegung]) a la luz de lo ya comprendido. De aquí se extrae que, debido a que la existencia es intrínsecamente hermenéutica, toda comprensión quedará signada por el carácter circular que es propio de todo proyectarse en el mundo. De este modo se plantea una definición importante: la circularidad es inherente a la comprensión en tanto existenciario, el volver sobre sí no representa una deficiencia en el comprender, sino su propio modo de ser.

\section{IMPLICANCIAS DE LA CIRCULARIDAD DE LA COMPRENSIÓN EN LA PRETENSIÓN CIENTÍFICA DE OBJETIVIDAD Y DEMOSTRACIÓN.}

Es en el contexto de la descripción del carácter circular de la comprensión que Heidegger comienza a discutir los conflictos que esta situación existencial genera en el ámbito de los saberes científicos tradicionales. En el parágrafo 32 de Ser y Tiempo, el problema del permanente retorno del comprender sobre sí mismo se plantea en su forma más expresa con ocasión del tratamiento de la cuestión del modo de conocimiento y demostrabilidad propios de las ciencias del espíritu, ${ }^{3}$ modelos de demostración que, no sólo a los ojos de las ciencias de la naturaleza, sino también ante los propios historiadores resultan insuficientes,

2 Desde luego esta descripción del movimiento de comprensión en términos de «salir y regresar» cumple una función exclusivamente ilustrativa que no debe confundirse con el verdadero proceso de la comprensión: La existencia es exterior de suyo, ella es en el modo de la relación con el mundo, y no debe superar ninguna barrera para realizar el tránsito de un hipotético ámbito interior (la conciencia) hacia uno exterior (el mundo en tanto «realidad»)

3 Sírvannos estas reflexiones para delinear una postura general referente a todas las ciencias ónticas, en lo que respecta a la compatibilidad entre la pretensión tradicional de cientificidad y la circularidad de la comprensión. 
e incluso falaces. Dejemos que Heidegger exprese claramente el conflicto que surge en las ciencias tradicionales al afirmar la circularidad de la comprensión:

El conocimiento científico pide el rigor del conocimiento evidenciante. La demostración científica no debe presuponer lo que ella tiene que demostrar. Pero si la interpretación debe moverse ya siempre en lo comprendido y nutrirse de ello, ¿cómo podrá producir resultados científicos sin moverse en un círculo, sobre todo si la comprensión presupuesta se basa, por otra parte, en el conocimiento ordinario del hombre y del mundo? (Heidegger 2006a, p.176)

En las lecciones de Marburgo del semestre de verano de 1925, recogidas en la obra Prolegómenos para una historia del concepto de tiempo se afirma que la incorporación del círculo en la comprensión de la historiografía es vivida incluso por los mismos historiadores como una falencia inevitable. El ideal del destierro de la subjetividad del investigador sigue dominando los estudios. Precisamente en el lamento ante la imposibilidad de semejante destierro se evidencia la dominación de una interpretación sesgada del comprender:

no es casualidad ni simplemente algo inoportuno que aparece en determinados intentos de entender en las disciplinas históricas el que se diga: «por desgracia hay algo que depende del punto de vista personal del historiador. No queda otro remedio que asumirlo, aunque el ideal sería que uno se pudiera librar de esa subjetividad». Tal opinión es absurda. El ideal es justamente que ese Dasein que está entendiendo forme parte del entender-de si mismo, y la consecuencia no es lamentarse de ello, sino ver ahí un quehacer, y poner el propio Dasein en el modo ocasional del entender-de para que, en cuanto entender-de, pueda procurarnos el acceso al asunto que se trata de entender (Heidegger 2007a, p.323)

Según estos dos fragmentos, pareciera que el carácter circular de la comprensión, y su basamento en el ordinario «saber» de habitualidad resulta pernicioso para las ciencias (naturales y del espíritu), en vistas a dos perjuicios principales mencionados al comienzo de nuestro estudio. Este es el sentido de circularidad contra el cual se manifiestan los científicos, en palabras de Kockelmans:

«La mayor parte de los lógicos adoptan una actitud negativa hacia el círculo como modo de pensamiento. Su actitud es completamente justificada, siempre y cuando nos limitemos a un círculo que ocurre en un argumento formal, o al círculo en la definición de un concepto.» (Kockelmans 1985, p. 85)

El primer modo de rechazo del carácter circular de la comprensión se expresa, en el caso de las ciencias históricas, en la insistencia en «no introducir nada en los textos» (Heidegger 2008, p.118). El análisis de las fuentes [Quellen] debe estar liberado de cualquier consideración subjetiva que sea 
aportada por el investigador. Tal como se expresa en el tratado de $1924 E l$ concepto de tiempo, -obra que analiza las posturas de Wilhelm Dilthey y el conde de Yorck en torno a la constitución de lo histórico- en el ámbito científico de la historiografía, se considera que reflexionar sobre lo traído de antemano provoca una perniciosa subjetivación de los resultados, claramente contrarios al ideal positivista de «objetividad». Sin embargo, tanto Yorck, como Dilthey, como sus posteriores, reconocen la imposibilidad de eliminar la implicación del investigador en la realidad a la que se dedica. Aquellos que afirman no introducir nada en los textos, aún incorporan (aunque sin percatarse de ello) las obviedades de la opinión pública.

Un permanente regreso sobre lo ya comprendido, representa, a los ojos de las ciencias positivas un círculo vicioso, una deficiencia en las reglas de la demostración, que impide la expansión y avance de un saber.

Ahora bien, según las más elementales reglas de la lógica, el círculo es un circulus vitiosus. Pero de esta manera el quehacer de la interpretación histórica queda excluido a priori del dominio del conocimiento riguroso. (Heidegger 2006a, p.176)

Ante esta crítica esgrimida contra las ciencias del espíritu, Heidegger opta por radicalizar la posición atacada: Siempre se posee de antemano lo comprendido, en cierto sentido. Sin un mínimo de noticia sobre aquello hacia lo que se dirige la búsqueda, el conocimiento no sabría por dónde comenzar su trayecto. Las ciencias del espíritu y de la naturaleza se esfuerzan en evitar la circularidad de sus demostraciones, pero en este esfuerzo pasan por alto la nada menos que la constitución esencial del comprender, y el carácter fundado del teorizar, en tanto conocimiento de objetos.

Sin embargo, ver en este círculo un circulus vitiosus y buscar cómo evitarlo, o por lo menos «sentirlo» como imperfección inevitable, significa malcomprender radicalmente el comprender. (Ibid.)

En Los conceptos fundamentales de la Metafísica, Heidegger se detiene en la apreciación que el entendimiento vulgar hace del círculo como modelo del comprender. Allí se afirma que el tipo de comprensión pre-filosófica sólo puede ver lo que se encuentra delante de ella, y procura siempre avanzar hacia la siguiente cosa, movimiento al que el entendimiento vulgar ha dado en llamar «progreso».

Pero como el progreso es el criterio de comprensión del entendimiento vulgar, toda marcha circular es de entrada una objeción, un indicio de imposibilidad. (Heidegger 2010, p. 237) 
De modo que el desafío, no únicamente para las ciencias del espíritu, sino para toda ciencia en general no consiste en encontrar el modo de escapar del círculo hermenéutico, sino más bien en precisar la manera de moverse en su interior desde una visión crítica de todo lo que hay de previo en el comprender. La vía para acceder críticamente al círculo se conquista por medio de la Analítica existencial: en lugar de extraer lo pre-comprendido (haber, ver y concebir previos) de creencias u opiniones populares, esto es, del estado público de interpretación (estado de la comprensión asociado al Uno [Das Man]) se vuelve imperioso asegurar su cientificidad, sometiendo lo pre-comprendido a examen para cerciorarse de que aquellas tres dimensiones previas han sido extraídas de una referencia a las cosas mismas; en palabras de Heidegger:

En él [en el círculo] se encierra una positiva posibilidad del conocimiento más originario, posibilidad que, sin embargo, sólo será asumida de manera auténtica cuando la interpretación haya comprendido que su primera, constante y última tarea consiste en no dejar que el haber previo, la manera previa de ver y la manera de entender previa le sean dados por simples ocurrencias y opiniones populares, sino en asegurarse el carácter científico del tema mediante la elaboración de esa estructura de prioridad a partir de las cosas mismas. (Heidegger 2006a, p.176)

Kockelmans afirma que, desde esta perspectiva, la función de la filosofía en su relación con las ciencias pasa a ser la de clarificar y dar fundamentación a la totalidad de presuposiciones que constituyen nuestra situación hermenéutica en cada caso ( $C f$. Kockelmans, 1985, p.87). La filosofía hace su aparición dentro del ámbito de las ciencias en la forma de la ontología regional, un estudio dirigido a la aclaración de los conceptos fundamentales [Grundbegriffe] de una ciencia, esto es, las nociones que funcionan como «hilos conductores» (Heidegger, Op.cit., p. 378) para la investigación de un ámbito del ente, una región ontológica. El objetivo de la ontología regional es el desarrollo de la ontología implícita que existe en el trasfondo de toda ciencia (Cf. Heidegger 2007c, p.180). La misma representa un cierto «despertar» (Cf. Heidegger 2000, p.83), un proceder incipientemente filosófico por medio del cual las ciencias cuestionan la constitución de sus hilos conductores, los conceptos fundamentales.

En su libro Heidegger's philosophy of science, Trish Glazebrook, con ocasión del tratamiento del problema del método experimental, reconoce que la noción de circularidad del comprender ha realizado un importante aporte a las concepciones más contemporáneas de la ciencia. A esta incorporación se debe la consideración de la imposibilidad de «meros hechos», ${ }^{4}(C f$. Glazebrook 2000, p.98) ya que toda interpretación (científica o no) de lo circundante pro-

4 Heidegger, por su parte, coloca a Galileo y Kepler como los primeros pensadores en haber divisado esta situación, en el contexto del desarrollo del proyecto matemático de la naturaleza. 
viene ya de un basamento de «saberes» pre-conceptuales, luego, toda ciencia incorpora los estados de cosas a los que atiende desde un sistema conceptual y proyectado de antemano. Ésta es también la opinión de John Caputo «esta intuición es uno de los más significativos puntos de contacto entre Heidegger y las recientes lecturas de la historia y filosofía de la ciencia. No hay hechos excepto dentro del horizonte pre-dado que les permite aparecer en primer lugar» (Caputo 1986, p.52)

Por lo visto, el intento de las ciencias de escapar al círculo hermenéutico resulta, en todos los casos, vano. Escapar al círculo del comprender equivale a renunciar a la estructura fundamental de este ente que somos nosotros mismos.

«El «círculo» en el comprender pertenece a la estructura del sentido, fenómeno que está enraizado en la estructura existencial del Dasein, en el comprender interpretante. El ente al que en cuanto estar-en-el-mundo le va su ser mismo, tiene una estructura ontológica circular» (Ibid.)

Al comprender le corresponde esencialmente un retornar sobre sí, sobre lo pre-comprendido. «La explicación interpretativa de fenómenos es sólo posible en la medida en que el que entiende trae con sigo desde su propio punto de vista una cierta precomprensión de este fenómeno y del contexto en que se manifiesta» (Kockelmans, 1985, p. 86 ) La pretensión científica de ausencia de prejuicios en la investigación resulta compatible con la circularidad del comprender: para certificar la rigurosidad, sólo hace falta asegurar la cientificidad de lo precomprendido, por medio de una pertinente apropiación fenomenológica de las cosas mismas. La verdadera lucha no es contra el carácter retornante de la comprensión, sino contra la fundación de lo pre-comprendido en el estado público de interpretación, que domina el vivir cotidiano. En efecto, las ciencias han de reflexionar sobre el hecho de que siempre se estudia desde algún «pre-» [vor-]. Es necesario que el horizonte de comprensibilidad sea sometido a crítica, procurando que éste haya provenido de las cosas mismas, y no de las opiniones y conjeturas de la publicidad [Öffentlichkeit].

Con ocasión de la consideración del círculo hermenéutico han hecho su aparición dos estructuras a las que deberemos atender: la triple configuración de la precomprensión y la estructura hermenéutica de todo comprender, la así llamada estructura del en tanto que [Als Struktur] (Cf. Tugendhat, 1970: 291). La primera estructura nos ofrecerá la explicación existencial del horizonte histórico del cual surge, y en el cual se instala toda ciencia; por su parte, la estructura $a l s,{ }^{5}$ será estudiada en tanto estructura que resulta modificada en el ingreso a la

5 De hecho, en la modificación de la estructura $A l s$ se encierra la posibilidad de la conversión de la mirada de acceso al mundo, que convierte al ente descubierto por la ocupación en el objeto de un contemplar teórico; esta conversión recibe el nombre de tematización [Thematizierung], y consiste, precisamente, en el traspaso del als hermenéutico (de la comprensión antepredicativa), 
actitud teórica. Comenzaremos, pues, por la aclaración de la noción de estado interpretativo y su relación con los niveles de la precomprensión, para luego estudiar el concepto de situación hermenéutica (en tanto modalización de la estructura $A l s$ ) y su papel fundamental en la definición de las tareas fácticas de una ciencia, de su carácter histórico y tradicional, de su posibilidad de crisis y desarrollo, y de su génesis existencial.

\section{El ESTADO INTERPRETATIVO Y LOS TRES NIVELES DE LA PRECOMPRENSIÓN}

El estado interpretativo constituye la configuración fáctica del haber previo, la forma previa de ver y la forma previa de concebir que funciona como trasfondo para cualquier modalidad de la interpretación. El despliegue fáctico de la existencia ya siempre se encuentra condicionado por un estado de interpretación que define de antemano el modo en que los fenómenos, entes y estados de cosas han de ser incorporados y articulados en el comprender del Dasein.

La vida fáctica se mueve en todo momento en un determinado estado de interpretación heredado, revisado o elaborado de nuevo [...] Estas perspectivas, que en la mayoría de los casos no están explícitamente disponibles y por las que la vida fáctica se desliza por la fuerza del hábito mucho mas que por la fuerza de una apropiación expresa, trazan las vías de realización por las que discurre la actividad del cuidado. (Heidegger 2002, p.37)

La comprensión puede estar asociada a la ocupación cotidiana, pero también puede asumirse a sí misma como tarea específica en la labor de investigación de una ciencia. El estado interpretativo opera como trasfondo para cualquier modo de la comprensión, o, más precisamente, para la etapa de articulación e incorporación explícita de lo comprendido. Esto significa: tanto la actitud teorética como la ateorética se encuentran definidas por un estado previo de interpretación que prefigura el modo en el que el mundo puede ser puesto al descubierto. ${ }^{6}$ «Las ciencias crecen en el marco de un inmediato estado interpretativo del Dasein y recaen -en sus resultados- en un semejante estado interpretativo» (Heidegger 2008, p. 53).

Puesto que la configuración previa de los tres vor- determina la manera en la que ha de ser interpretado un modo de ser en el mundo (tanto desde de la actitud cotidiana como desde la teorética) junto con el consiguiente descubrimiento de entes y estados de cosas, al estado interpretativo se lo denomina

al als apofántico (del enunciado que hace del ente el tema de un discurso)

6 De hecho, en Ser y Tiempo, la génesis existencial de la ciencia es descripta como la apertura de un a priori, (Cf. Heidegger 2006a:378) esto es, una forma previa de concebir y proyectar la constitución de ser de una región ontológica. Este a priori determina de antemano como qué debe ser comprendido un ente para permitírsele el ingreso a una determinada disciplina. 
también situación hermenéutica [hermeneutische Situation]. Un análisis de la situación hermenéutica consiste en la explicitación del modo de configuración de cada uno de los niveles de la precomprensión. ${ }^{7}$

No sólo el interpretar que desarrolla y custodia la circunspección del Dasein cotidiano, sino todo interpretar -incluido el del conocimiento explícitamente historiográfico- está determinado por los momentos estructurales indicados anteriormente, a saber: el haber-previo, la manera previa de ver y la manera previa de entender. Estos momentos constituyen la respectiva situación hermenéutica en la que cada interpretación tiene su posibilidad de ser. (Ibid., p.118)

Sobre el trabajo de análisis de la situación hermenéutica, comenta J.A. Escudero:

«La apropiación de la situación hermenéutica, de la que Heidegger habla en repetidas ocasiones, va encaminada a conquistar críticamente el horizonte de sentido ya siempre dado en el que se encuentra el fenómeno de la vida para desenmascarar, desmontar o destruir los conceptos operativos en la precomprensión de ese fenómeno de la vida y devolverlos a su origen». (Nota aclaratoria no4 de J.A.Escudero en: Heidegger 2002, p.87)

Los elementos constitutivos de la situación hermenéutica cumplen una función que ya fue mencionada, pero aún no profundizada: en ellos se muestra la operatividad de la estructura Als; estos principios predisponen a la comprensión de un fenómeno para acceder a él desde una determinada perspectiva. Los tres niveles de la precomprensión le indican a la comprensión en tanto qué ha de ser interpretado algo, o bien, de qué modo ha de ser estudiado este fenómeno, situación o estado de cosas; o también qué aspecto ha de ser considerado como prioritario o relevante, y cuáles deben ser postergados como prescindibles en la comprensión de un fenómeno.

La situación hermenéutica se articula en una serie de configuraciones concretas de la estructura Als, que vienen a determinar Cómo qué (en tanto qué) debe ser interpretado algo. Desde luego, la situación hermenéutica no es transparente para el Dasein que se despliega en el modo de la ciencia o de la ocupación pragmática cotidiana, sino que opera como un horizonte para la comprensión, es decir, inmediata y regularmente el Dasein no tiene una conciencia explícita del estado de interpretación, no se percata de la forma manifiesta de las configuraciones previas que matizan su interpretar.

7 La clarificación de la situación hermenéutica representa precisamente el plan fundamental del célebre informe Natorp. Esta obra busca desocultar las condiciones de comprensibilidad que operan en el trasfondo de la filosofía de Aristóteles. 
Estos «como algo», a partir de los cuales se interpreta el mundo circundante y la ocupación absorbida en él, regularmente no son descubiertos por primera vez por el respectivo Dasein. El Dasein, en cuanto convivencia, ha nacido y crecido en este permanente estado interpretativo. (Heidegger 2008, pp.111-112)

En la medida en que la configuración del estado de interpretación no depende de un originario descubrimiento a cargo del Dasein en su contacto con las cosas mismas, ni de una crítica sobre qué es lo que hay de previo y heredado en el comprender, sino que proviene de una tradición de opiniones surgida del mediano intercambio de la convivencia [Mitdasein], de consideraciones generales acerca del mundo, que aparecen en la forma de juicios carentes de autor que son propiedad de todos, y por lo mismo de nadie, opiniones difundidas acerca de lo que se dice y se hace acerca del mundo y del ente; en la medida en que la estructuración de la precomprensión proviene de este fondo vago y universal, es decir, del Uno [das Man] al estado de interpretación se lo denomina estado interpretativo público.

«El estado interpretativo público del Dasein guía el respectivo hablar sobre algo. En efecto, el estado interpretativo es el estado interpretativo de «Un tiempo», de «Una época». Lo que en «nuestro tiempo» se piensa sobre ... , a qué posibilidades del Dasein se da preferencia, cómo se concibe el Dasein mismo, lo que «flota en el ambiente», todo esto determina por termino medio las pretensiones, las necesidades y las empresas del Dasein». (Ibid., p.112)

Como pronto veremos, la noción de estado interpretativo es de relevancia fundamental para la descripción de la constitución de los saberes científicos, ya que toda disciplina teórica se realiza desde este estado público de interpretación. ${ }^{8}$

En este estado interpretativo radica el carácter tradicional de la comprensión, y también de la ciencia, como uno de sus modos. Toda interpretación se inscribe (sabiéndolo o no) en una genealogía de interpretaciones socialmente constituidas, de las cuales el Dasein fáctico no es enteramente responsable.

Este estado interpretativo ya ha decidido en cada caso que hay que cultivar y que hay que tratar en las diferentes posibilidades de la ocupación (la materia de la poesía, los temas del arte figurativo, los ámbitos de investigación de las disciplinas científicas) (Heidegger, 2008., p.113)

8 Este estado hermenéutico público rige en los inicios de toda ciencia, pero su influencia puede extenderse hasta un período muy avanzado de su desarrollo histórico. 


\section{EL HORIZONTE DE LA PRECOMPRENSIÓN Y LA CONSTITUCIÓN DE LOS SABERES CIENTÍFICOS}

Es en el sistema de pre-comprensión hasta aquí descripto donde puede rastrearse el ámbito de radicación existencial de la fase tradicional de la ciencia y sus tareas especializadas. La ciencia modaliza de manera particular aquellas tres dimensiones del sentido. El horizonte de comprensibilidad que define los comportamientos asociados al teorizar es un ámbito que, progresivamente -y siempre partiendo de una experiencia antepredicativa del mundo- va quedando constituido por sistemas, leyes y principios, por axiomas y paradigmas, por hipótesis y demostraciones, por artefactos, experimentos y observaciones, en otras palabras, el horizonte mismo se configura como teórico y tematizado. Si bien, indefectiblemente, la ciencia es tributaria de un saber de familiaridad, surgido del cotidiano ser-en-el-mundo, la configuración de la precomprensión que más específicamente define al teorizar se configura en su mismo proceso de desarrollo (en un movimiento sin dudas circular) por los resultados de las tareas científicas de investigación. Esta afirmación se hará más clara de inmediato: con ocasión de la explicación de la manera en la que las tres coordenadas de la precomprensión se configuran para el caso puntual de la historiografía, podemos observar en qué puede consistir la estructuración de la situación hermenéutica de una ciencia óntica en general:

Según que la situación hermenéutica sea aprehendida originariamente o simplemente aceptada tal cual, la investigación historiográfica tiene asegurada: 1) como qué se debe concebir desde el inicio el Dasein pasado (como fenómeno que expresa una cultura, como persona, como cosa insertada en el nexo causal de los acontecimientos), 2) desde qué perspectiva se interroga al objeto concebido anticipadamente de esta manera y 3 ) de que conceptualidad se dispone para una apropiación comprensora. Sin embargo, inmediata y regularmente la situación hermenéutica permanece inexpresada. (Heidegger 2008, p.118)

Como puede verse en este análisis puntual, el haber previo (primer componente del horizonte de comprensibilidad previa) de las labores fácticas de la ciencia está constituido por la definición (primero inexplícita y general) del como qué debe ser comprendido en ente al cual se refiere la investigación. El haber previo responde por anticipado a la pregunta sobre qué es aquel ente o región ontológica que funciona como objeto de estudio. La determinación $a$ priori de qué es aquello que ha de pertenecer a un ente para ser considerado miembro de una región ontológica, constituye un movimiento que se concretiza en el así llamado proyecto de la constitución del ser de una región ontológica. ${ }^{9}$

9 El caso más consagrado de este proyecto previo de la constitución de ser de una región es 
Este movimiento, que se cumple en el fenómeno a veces llamado tematización [Thematizierung] (Ser y Tiempo), y otras veces objetivación [Vergegenständlichung] (Interpretación fenomenológica de la Crítica de la razón pura de Kant), constituye, según las diversas exposiciones de Heidegger en el período de Marburgo, el proceso donde ha de buscarse el verdadero origen ontológico de una ciencia. Se trata de un movimiento que (primero de manera ingenua y general) realiza una demarcación de una región ontológica, y selecciona una serie de conceptos fundamentales, ${ }^{10}$ que funcionan como hilos conductores que penetran a todos los entes de la región, y orientan el estudio de los mismos.

Ciertamente, la definición previa del objeto de estudio (posibilitada por el previo proyecto de la constitución de ser de una región ontológica) viene determinada por las adquisiciones sedimentadas, conformadas por una serie articulada de de proposiciones, entes, instrumentos y saberes tematizados, socialmente constituidos y permanentemente disponibles v.gr.: la teoría ondulatoria de la luz, la brújula, la supernova, las leyes de la termodinámica, o bien, desde la perspectiva de las ciencias del espíritu, la idea de familia, de pulsión, de movilidad social, de grupos de poder etc. En Introducción a la investigación fenomenológica Heidegger define el haber previo como «lo que desde el comienzo se tiene para la investigación; aquello en lo que la mirada se detiene de continuo». (Heidegger 2006b, p.118). Por su parte, en Prolegómenos para una historia del concepto de tiempo, se explica el haber previo como aquello gracias a lo cual la interpretación ya entiende del de-qué ha de hablar el discurso; es la predeterminación de aquello sobre lo cual el discurso va a discurrir (Cf. Heidegger 2007a, p.375).

El modo previo de ver (segundo componente del horizonte de comprensibilidad previa) queda definido por el punto de vista desde el cual el mundo, en tanto objeto de estudio es interrogado, «Aquello sobre lo cual se tiene a la vista lo situado en el haber previo, según lo cual se lo ve, con respecto a lo cual viene a estar a la vista es lo que denominamos mira previa» (Heidegger 2007a, p.375). Este punto de vista sugiere qué cuestiones caben ser preguntadas, que aspectos ameritan ser observados y cuáles exceden el ámbito de la ciencia para precipitarse hacia el de lo no-científico. Los artefactos, experimentos, e investigaciones surgen de un previo punto de vista que dictamina en qué dirección debe interrogarse al mundo, cuáles aspectos deben ser tenidos en cuenta, y cuáles carecen de relevancia. Este punto de vista se cristaliza y articula en la configuración fáctica de los tes Vor-, pero viene determinado desde su origen por

el así llamado proyecto matemático de la naturaleza, el cual abre de antemano al ente únicamente en su dimensión calculable.

10 En el caso de las ciencias de la naturaleza, por ejemplo, los conceptos fundamentales serían las nociones de tiempo, espacio, masa, movimiento, etc. 
el proyecto de la comprensión del ser, que diera origen a una región ontológica. Ciertamente no puede haber descubrimiento de objetos que se encuentre libre de contexto; la investigación científica ocurre dentro de un plano [Grundriss] que determina las condiciones de relevancia (Cf. Guignon 1986, p.159).

El modo previo de concebir (tercer componente del horizonte de comprensibilidad previa) está integrado por el complejo armazón conceptual en el cual las tareas de la ciencia adquieren sentido y funcionalidad, siempre en función del previo proyecto de la comprensión del ser. La Vorgriff, indica de antemano «los nexos de significación que se van a llevar a concepto en el discurso interpretativo y en particular en el discurso científico.» (Heidegger Op.cit., p.375) Lo que parece olvidarse en la actitud teorética es que los entes necesitan ser «trabajados» [Bearbeitung] para encajar en los modelos explicativos ( $C f$. Guignon, 1986, p.159) Entre las concepciones previas encontramos decisiones históricas de tipo metodológico, definiciones de objetos o procesos, e incluso concepciones de la verdad o probabilidad. Sólo por mediación de una teoría puede un especialista afirmar, por ejemplo, que la aparición de un punto en el radar representa la presencia de un objeto en las cercanías.

Los conceptos, artefactos, prácticas y entes que vertebran a la ciencia (en su sentido lógico de entramado de enunciados articulados por relaciones de fundamentación) no son transparentes en su relación con el mundo, sino que su primacía y utilidad viene decidida de antemano por el estado de interpretación. En este contexto, el papel de la ciencia será el de avanzar hacia nuevos temas o profundizar en las cuestiones canónicas, y no el de cuestionarse la genealogía de su horizonte de comprensión, ni poner en duda el origen de aquello que determina su haber, ver y concebir previos; como ya se ha visto, semejante tarea de cuestionamiento de la situación hermenéutica constituye un movimiento incipientemente filosófico. Las ciencias no realizan un regreso sobre sus propias condiciones de comprensibilidad, mientras los entes y fenómenos a los que se dedican respondan de forma satisfactoria ante los cuestionamientos que ella realiza, siendo vistos desde un punto de vista y vía de acceso definidos de antemano (en un proyecto previo de la comprensión de una región). Sólo cuando el ente se muestra resistente, por ejemplo, cuando se detecta un nuevo tipo de fenómeno para el cual las categorías usuales no son suficientes, debe la ciencia realizar una autocrítica, y observar cuál era efectivamente el contenido de su estructura de precomprensión. Por supuesto, al hacer este regreso sobre sí misma, la ciencia obtendrá una insustituible ayuda en la ontología regional cuyo objeto de estudio coincide con el de la ciencia en cuestión.

Mientras el contacto con el ente no ponga en jaque la estructura de la precomprensión, la tarea de la ciencia será la de proteger los conceptos fundamentales y ampliar el alcance de las teorías. Esta actitud es denominada por Heidegger custodia constitutiva de los conceptos. 
Lo que antes estaba abierto de manera originaria se encuentra ahora en la modalidad de custodia constitutiva de los conceptos y de los principios. Las verdades se convierten en «valideces» suspendidas en el aire. La posesión de lo que es universalmente válido hace que ya no sea necesario volver a preguntarse por los originarios nexos ontológicos que constituyen el terreno sobre el que se basa la ciencia.(Heidegger 2008, p.54)

\section{Conclusiones}

Heidegger afirma que el verdadero avance de una ciencia no está dado por los nuevos descubrimientos, por la acumulación de casos, ni por la ampliación de sus proposiciones hacia nuevos ámbitos, sino por la capacidad de experimentar una crisis en sus conceptos fundamentales $(C f$. Heidegger 2006a, p.35). Pues bien, ahora puede añadirse una precisión a aquella afirmación: la transformación en la que se juega el desarrollo de una ciencia consiste en una revisión y modificación de la situación hermenéutica, esto es, en un examen de la manera previa de ver y de preguntar; una reflexión sobre el modo de acceder al ente y sobre la forma en que el ente es abierto de antemano para permitir su investigación. Al respecto, se afirma en Los conceptos fundamentales de la Metafísica:

Esta transformación del ver y del preguntar es siempre lo decisivo en la ciencia. La grandeza de una ciencia y su vitalidad se ponen de manifiesto en la fuerza para la capacidad de esta transformación. (Heidegger 2010, p.315)

Sobre el papel destacado de la revisión de los conceptos fundamentales para la comprensión del origen ontológico de una ciencia, Heidegger expresa en Ser y tiempo:

Con la elaboración de los conceptos fundamentales de la conductora comprensión del ser se determinan los hilos conductores de los métodos, la estructura del aparato conceptual, la correspondiente posibilidad de verdad y certeza, el modo de fundamentación y demostración, la modalidad del carácter vinculativo y el modo de la comunicación. El conjunto de estos momentos constituye el concepto existencial plenario de la ciencia. (Heidegger 2006a, p.378)

Como indica este fragmento, la crisis y el avance se experimentan en el cuestionamiento de los tres niveles previos de la comprensión. De hecho, afirma Heidegger, en esta capacidad de retornar sobre sí misma -en la que se funda su auténtico avance- radica el carácter histórico de toda ciencia.

«La zoología, y así toda ciencia, es histórica, no solo en sus progresos en los resultados, sino igualmente en sus retrocesos en el modo de su preguntar. Los retrocesos de una ciencia son la mayoría de las veces inaparentes, pero son más centrales que los progresos. [...] Toda ciencia es histórica porque cambia 
y se modifica en la postura fundamental de la ciencia respecto de su ámbito, en la concepción del ámbito en general» (Heidegger 2010, p.238).

Como puede verse, el terreno de ocurrencia de una crisis, y, por tanto, de desarrollo y profundización de una ciencia es el delineado por la situación hermenéutica. El decisivo avance de una ciencia se da en la revisión de los tres niveles de la precomprensión. Ahora bien, la relevancia del horizonte de precomprensión en la caracterización ontológica de una ciencia no se agota en la indicación de su papel en tanto contenido que resulta sometido a crítica en un proceso de crisis, sino que se extiende a niveles más fundamentales.

En primer lugar, la génesis ontológica de una ciencia se juega en la determinación (primero ingenua y general) de los conceptos fundamentales, de los objetos de estudio, de los modos de preguntar y de acceder al ámbito de estudio, $\mathrm{y}$, por supuesto de la consiguiente pre-selección de lo relevante e irrelevante en este modo de acceso. En otras palabras, la triple dimensión de la precomprensión juega un papel fundamental en el origen ontológico de una ciencia.

En segundo lugar, la circularidad del comprender, fundada en los tres niveles previos de la comprensión, también se muestra como la condición existencial de posibilidad del carácter tradicional e histórico de una ciencia (desde luego, una condición cooriginaria con la historicidad del Dasein).

Nuestra reconstrucción de las reflexiones heideggerianas del período de Marburgo nos ha mostrado el modo en que la ciencia ha de convivir con la dimensión circular de toda comprensión, y ha explicitado el motivo por el cual la misma no ha de percibirse como una falencia en el desarrollo de las investigaciones, ni como un obstáculo a ser evitado.

Hasta este punto, hemos intentado -conforme a nuestro objetivo inicialponer de manifiesto las particularidades de cada una de las tres dimensiones de la precomprensión, para indicar, por medio de una articulación de temáticas dispersas a lo largo del período de Marburgo, el modo en que el triple horizonte de sentido define no sólo las tareas fácticas de una ciencia, sino también su carácter histórico y tradicional, su posibilidad de crisis y desarrollo, y su misma génesis existencial.

\section{REFERENCIAS BIBLIOGRÁFICAS:}

HEIDEGGER, M. (1995) Phänomenologische interpretation von kants Kritik der Reinen Vernunft, , Frankfurt am Main: Vittorio Klostermann (GA 25)

, (2000): Los problemas fundamentales de la fenomenología, Madrid: Trotta, Trad. y prólogo de Juan José García Norro.

___ (2002): Interpretaciones fenomenológicas sobre Aristóteles, indicación de la 
situación hermenéutica [informe Natorp] Madrid: Trotta. Trad. de Jesús Adrián Escudero

____, (2004): Lógica: la pregunta por la verdad, Madrid: Alianza. Versión española de J. Alberto Ciria.

___ (2006a) Ser y Tiempo, Madrid: Trotta. Traducción y notas de Jorge Eduardo Rivera.

___ (2006b) Introducción a la Investigación Fenomenológica. Madrid: Síntesis. Traducción de Juan José García Norro.

____ (2007a) Prolegómenos para una historia del concepto de tiempo, Madrid: Alianza. Traducción de Jaime Aspiunza

___ (2007b) Principios metafísicos de la lógica, Madrid: Síntesis. Traducción de Juan José García Norro

___ (2007c) Seminarios de Zollikon: protocolos, diálogos, cartas. México: Jitanjáfora $\mathrm{M}^{\mathrm{o}}$ Relia Ed. Trad. De Ángel Xolocotzi Yáñez

___ (2008) El concepto de tiempo, Barcelona: Herder, Traducción de Jesús Adrián Escudero.

, (2009) Tiempo e Historia, Madrid: Trotta. Edición y traducción de Jesús Adrián Escudero

___ (2010) Los conceptos fundamentales de la metafísica: Mundo, finitud, soledad». Madrid: Alianza, Traducción de Alberto Ciria

CAPUTO, John D.: (1986) «Heidegger's Philosophy of Science: The Two Essences of Science.» En Rationality, Relativism and the Human Sciences, ed. J. Margolis, M.Krausz, and R. M. Burian. Dordrecht: Martinus Nijhoff

, (2000), «Hermeneutics and the natural sciences : Heidegger, science,and essentialism». en: John D. Caputo: More radical hermeneutics : on notknowing who we are. Bloomington, Ind.: Indiana Univ. Press, pp. 151 -172

DREYFUS, H.: (1996): Ser en el mundo, comentario a la división I de Ser y Tiempo, Chile: Cuatro vientos. Traducción Francisco Huneeus.

ESCUDERO, J.A (2011) Heidegger y la genealogía de la pregunta por el ser, Barcelona: Herder

GETHMANN, K. F. (1974) Verstehen und Auslegung, Bonn, Bouvier , (1991) „Der existenziale Begriff der Wissenschaft. Zu Sein und Zeit, § 69b« en

GETHMANN, K. F (Hrsg.) Lebenswelt und Wissenschaft. Studien zum Verhältnis von Phänomenologie und Wissenschaftstheorie, Bonn, Bouvier

GLAZEBROOK, T. (2000): Heidegger's philosophy of science, New York: Fordham University press.

GUIGNON, CH. (1983): Heidegger and the problem of knowledge, Indiana, USA: Hackett publishing company

KOCKELMANS, J.J. (1985): Heidegger and science, Washington D.C.:Center for advanced research in phenomenology \& University press of America 
NEUMANN, G. (1999) Die phänomenologische Frage nach dem Ursprung der mathematisch-naturwissenschaftlichen Rauauffassung bei Husserl und Heidegger, Berlin, Duncker \& Humboldt.

TUGENDHAT, E. (1970) Der Warheitsbegriff bei Husserl und Heidegger, Berlin: Walter de Gruyter \& Co.

LuCIANO MASCARÓ ES PROFESOR ADJUNTO en la materia «Metafísica» En la Universidad de Ciencias Empresariales y Sociales (UCES) y Profesor adjunto en la materia «Filosofía del Lenguaje» En la Universidad Católica Argentina (UCA).

Línea de investigación:

Hermenéutica, Fenomenología hermenéutica, Filosofía de la tecnología

Publicaciones:

(2016). «El carácter artefactual del enunciado en la filosofía heideggeriana de los años '20: un análisis crítico de las interpretaciones de Rainer Bast y Robert Brandom». En Pensamiento: revista de investigación e información filosófica, pp. 119-129.

(2015). «El concepto lógico de la ciencia en la filosofía heideggeriana del período de Marburgo: en busca de una caracterización integradora del enunciado científico y de sus condiciones de posibilidad» En Eikasía, revista de Filosofía, pp. 325-354

Correo electrónico: lcnmascaro@hotmail.com 